\title{
CURRENT STATUS OF HM SgE MEASUREMENTS
}

\author{
H. E. Matthews and W. A. Sherwood \\ Max-Planck-Institut für Radioastronomie \\ Auf dem Hügel 69,5300 Bonn \\ Federal Republic of Germany
}

A summary of radio and infrared observations of HM Sge made between late May and September $1^{\text {st }}, 1977$, is given.

Radio. HM Sge was observed by W. A. Altenhoff on 28 . May 1977 at $15 \mathrm{GHz}$ with the Effelsberg $100 \mathrm{~m}$ telescope. The flux density was $41 \pm 6 \mathrm{mJy}$. This agrees with the observation by Feldman (IAU CIRC. 3083) on 19. May at $10.7 \mathrm{GHz}$. HM Sge seems to be optically thin at frequencies above $10.7 \mathrm{GHz}$.

Measurements made with the assistance of T. A. Th. Spoelstra at Westerbork at $1.4 \mathrm{GHz}$ since late May (Figure 1) show that the flux density is decreasing overall. Monitoring is continuing and as this goes to press the flux density continues to decrease. The calibration was checked against the nearby source $O V+168$. No confusing sources appear to be in the single dish beams. Note the steep decrease near day 210.

Infrared. HM Sge was observed by E. Kreysa, G. V. Schultz, W. A. Sherwood and H. Tovmassian from $1.25 \mu$ to $3.6 \mu$ between 2. and 7. July 1977 at Byurakan observatory with the $2.6 \mathrm{~m}$ telescope and from $1.25 \mu$ to $30 \mu$ between 18. August and 1. September at ESO La Silla with the $1 \mathrm{~m}$ telescope. Although in the visual HM Sge brightened by $0 .^{\mathrm{m}} 5$ (IAU CIRC. 3094; Ciatti, private communication) there was no change in the near infrared greater than $0 .{ }^{\mathrm{m}} 1$ (and probably less than 0.05 ). The combined data is shown in Figure 2. The steep spectrum is similar to that found for V 1016 Cyg. 


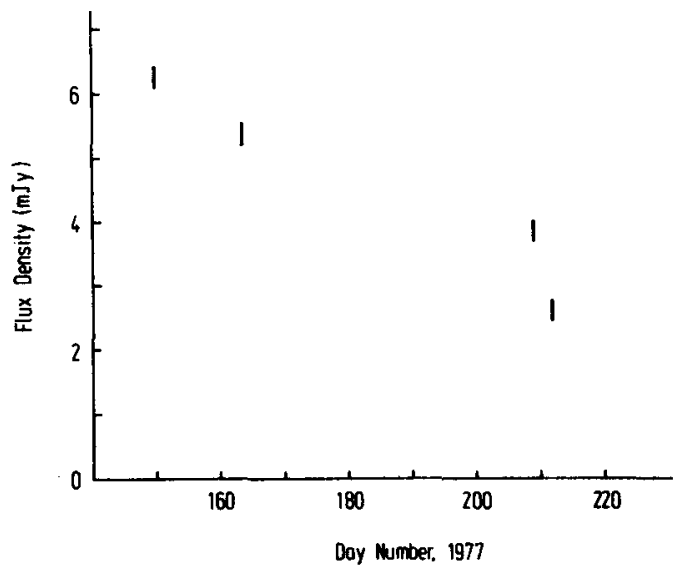

Fig. 1. The behaviour of $1414 \mathrm{MHz}$ flux density of HM Sge as a function of time.

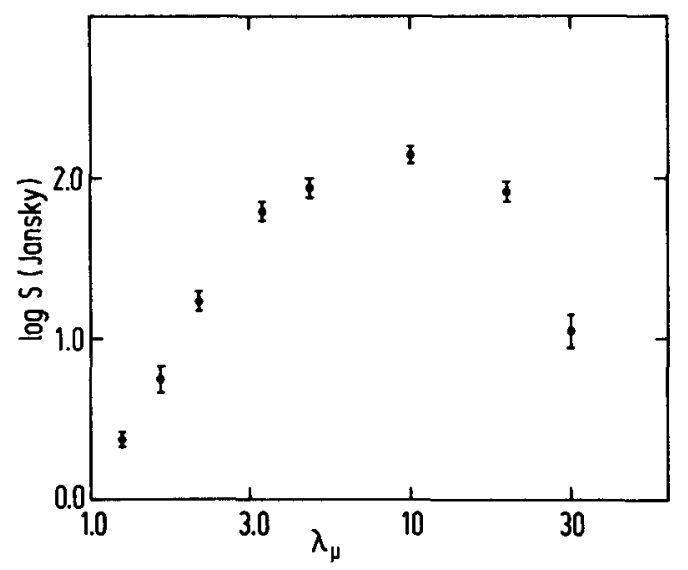

Fig. 2. The infrared flux density distribution between $1.25 \mu$ and $30 \mu$. 